\title{
Covid-19 and E- consultation in Orthopaedics: Experience on delineating the spectrum of Orthopaedic disorders amenable to treatment via Tele-consultation
}

Purushotham Lingaiah ( $\sim$ puru@aiimsmangalagiri.edu.in )

All India Institute of Medical Sciences

Venkateshwar Reddy

All India Institute of Medical Sciences

\section{Method Article}

Keywords: Tele-consultation, Orthopaedics, Covid-19

Posted Date: September 29th, 2020

DOl: https://doi.org/10.21203/rs.3.rs-83359/v1

License: (c) (1) This work is licensed under a Creative Commons Attribution 4.0 International License. Read Full License 


\section{Abstract}

Context: Covid-19 pandemic and lockdown scenario has forced to shut down the Outpatient consultation in hospitals and clinics. It was challenging to help out the patient population who were routine health care seekers for maintenance and follow-up.

Aims: To tackle the prevailing routine Orthopaedic health care issue due to Covid-19 lockdown strategy through Tele-consultation and to delineate the spectrum of Orthopaedic disorders that were amenable to treatment by e-Consultation.

Methods and Material: We executed two Tele consultation designs (Call based and App based) for our institution to reach out to the patients. The Orthopaedic patient characteristics and disease management was documented and analyzed.

Results: A total of 468 consultations with diverse Orthopaedic disorders including Congenital lesions, Developmental and Metabolic disorders, Infective disorders, inflammatory disorders, Traumatic disorders, Tumours, Degenerative disorders and Repetitive Strain Injuries were addressed.

Conclusions: Clinical decision making through Tele-consultation in Orthopaedics is demanding. However, majority of routine Orthopaedic disorders are amenable to management.

\section{Introduction}

In the scenario of Covid-19 pandemic and lockdown, Tele-consultation that was once considered experimental and futuristic has now become a reality ${ }^{1}$. The practice got legitimized by the Government of India new "Tele medicine guidelines" for remote Tele consultations ${ }^{2}$. Orthopaedics is one of the majorly consulted departments in any hospital in normal situations ${ }^{3}$. The burden of orthopaedic disorders compels the patients to inactivity and increases the morbidity resulting in loss of days at work. During lockdown, all health institutions were forced to shut down their non essential inpatient and outpatient facilities lasting more than 42 days. Routine Orthopaedic health care seekers were distressed for their maintenance and follow up visits for outdoor consultation. Helping out this patient population, who were unable to visit the hospitals became a challenge.

Though Tele orthopaedics has evolved as a major modality of treatment elsewhere, it is still under trial and scrutiny in India 4 . Video consultation has gained importance in recent times ${ }^{5,6}$. In order to tackle the prevailing routine health care issue due to Covid-19 lockdown strategy, we executed two Tele consultation designs for our institution to reach out to the patients. In this study, we share our experience of providing e-consultation to the patients in need and also delineate the spectrum of Orthopaedic disorders that were amenable to treatment by e-Consultation.

\section{Methods}


The Tele consultation service at our Institute was started with effect from 15th April 2020. The service was named e-Paramarsh. We launched two Tele-consultation modalities simultaneously.

In the First approach, we designated Orthopaedics department specific telephone number on tablets kept in OPD. Applications of WhatsApp video and audio calls were activated on these devices. Patients were given a time slot (09:00 AM - 11:00AM) for registration. Registration process was as follows: Patients would call the phone number of the Department for Registration. After the call is connected, basic details (Name, Age, Sex, Father Name, Address, WhatsApp Phone Number and Chief Complaints) were asked and noted. Patients were informed that they would receive a call from the Doctor after 11:00AM. The registered patients list was passed on to the Doctors. Doctors would call back the patient through Video call/ Audio call / Normal call. After consultation, the doctor's prescription was scanned and sent through WhatsApp to the patient's mobile phone. In case WhatsApp was not available with the patient, a text message prescription was sent (fig 1).

In the second approach, an App (AllMS Mangalagiri e-Paramarsh) was developed by CDAC (Centre for development of advanced computing) and activated for consultation by the patients. In this approach patients were required to download the e- Paramarsh App (CDAC Ltd India) available free of cost in their android phone. Once activated, patients were required to log in on the app through the patient login portal by entering the personal phone number which would be authenticated by an OTP. The registration would now start by selecting the Orthopaedics department and filling of basic details similar to the details that were asked in Call based registrations. A column to enter the chief complaints was also provided. Doctor would then login with Doctor Login ID of the institute, accept the registration requisition raised by the patient and then call back through the App. e-Prescription would be generated by entering the details on the prescription page. After confirmation by the doctor, the prescription was sent to the patient for download through the same App (fig 2). Tele consultation services was provided free of cost to all patients in both the modalities.

Our hospital made provisions for mobile investigation (doorstep blood sample collection) to those requiring blood tests. The patients requiring detailed examination and/or emergency care were called to OPD for Consultation after a thorough triaging for Covid-19.

The registrations were divided into three categories for the purpose of data collection and analysis. New cases included those who consulted the doctor through Tele-consultation for the first time. Old cases included those who had consulted face-to-face prior to Covid-19 outbreak and the follow-up consultation was done via Tele-consultation. Follow-up cases included both new and old cases registered for their subsequent consultations via Tele-consultation.

The details of patient's including demographic data, complaints, diagnosis and management were electronically recorded in Hospital information management system of the institute.

\section{Results}


Data from 15th Apr to 14th Aug was compiled and analyzed. A total of 478 registrations for Orthopaedics Tele-consultation were received during this period. Out of these 478 registrations, 10 consultations were labeled as missed due either to non availability of network or to non responsiveness to call back from doctors. These 10 registrations were excluded and hence 468 case registrations were included in the study. The cases were divided into New cases, Old cases and follow-up cases. (Fig 3)

The demographic details and patient characteristics are described in table 1.

The diagnosis was classified into following categories (Table 2): Congenital lesions, Developmental and Metabolic disorders, Infective disorders, inflammatory disorders, Traumatic disorders (chart 1), Tumors, Degenerative disorders and Repetitive Strain Injuries (RSI). This categorization covers almost all Orthopaedic disorders.

The treatment of patients through E-consultation was based on the basic principles of Orthopaedic disease management. Clinical examination, Lifestyle modifications and physiotherapy were innovatively demonstrated to the patients. The details are available in the section on discussion.

\section{Discussion}

Tele-consultation is a general term for any consultation between doctors or between doctor and patients on a network or video link (e.g., Face time, intranet, Internet, Skype, etc.) $)^{7}$. The primary goal of Teleconsultation is to bridge the geographical gaps for easy access to standardized health care. The goal can be extended to include the current scenario of Corona virus pandemic wherein the access to health care facility for routine check-up is significantly hampered due to the lockdown imposed to control the spread of Covid-19 infection. Tele-consultation is helping in maintaining the social distance and thus is playing a vital role in safe and efficient health care delivery. The World Health Organization mentioned Telemedicine among essential services in "Strengthening the Health Systems Response to COVID-19" policy ${ }^{8}$.

On 11th March 2020, in an attempt to mitigate COVID-19, the functioning of all major and minor services came to a standstill as the country announced complete lockdown. This meant that even the outpatient department which is often considered as Shop window of the hospital was not accessible anymore ${ }^{9}$. Attending to the medical needs of the patients became challenging and hence telemedicine was resorted to that provided an opportunity to both the patient and the doctors to have a virtual conversations thereby helping resolve the health concerns of non-serious patients.

The patient characteristics in our study were far from different to any Orthopaedic Outpatient department. Diagnosis and management though appeared distinct, the principles remained the same.

Congenital disorders - Paediatric Orthopaedics is a less commonly practiced specialty in telemedicine as most parents wish to get a face-to-face consultation with the physician for their children ${ }^{10}$. Congenital disorder being a part of Paediatric Orthopaedics was a great challenge to manage. We received one case 
of 2 month old baby with Bilateral Congenital Talepes Equino Varus (CTEV). A video call with the parents was done. The virtual examination of the baby primarily to rule out Syndromic Clubfoot was done. This was aided by a medical student who was a resident of the same apartment. Considering the health and immune status of the mother and the baby, hospital visit for Ponseti technique of clubfoot correction was deferred. However, the mother was clearly explained about the condition and the importance of manipulation of the foot. Four to five sessions of manipulations were monitored through Video call. A YouTube link was also provided to the parents to continue practicing the manipulation ${ }^{11}$. The child was eventually taken to the clinic for further care after 3 months when access to the hospital was feasible. Virtual Clubfoot clinic at Pandemic situations like Covid-19 can be of great help. The function of such a clinic shall include monitoring the procedure of manipulation, look for progress of correction and importantly remotely visualize the complications of plaster.

Developmental and Metabolic - Rickets is one of the most commonly encountered metabolic disorders in children. Four cases were seen through Tele-consultation. Classical features of widened wrist, Bowlegs, Frontal Bossing and Pectus Carinatum were seen in these cases which made the clinical diagnosis easy. Oral Vit-D was advised. Though Outdoor activities were completely shut down during the pandemic, parents were instructed to make arrangements for adequate sun exposure.

Inflammatory disorders - Rheumatoid arthritis (RA) is one of the most common inflammatory diseases with a prevalence of $0.9 \%$. Out of 54 cases of inflammatory diseases, 53 cases were RA and 1 was Ankylosing spondylitis. 41 cases were already on treatment and 12 were newly diagnosed. Though various guidelines for DMARD therapy has been introduced, the treatment protocol still varies from centre to centre. In most cases of naive RA, combination DMARD is prescribed. This combination most likely consisted of Methotraxate (MTX) + Hydroxychloroquine (HCQ) (27), MTX + Sulfasalazine (SAAZ) (5) or HCQ + SAAZ (1). Due to sudden increase in requirement of HCQ for the prophylaxis of Covid-19, the source of supply was exhausted and patients who were already on DMARD combination therapy with HCQ had to be shifted to a different Combination. HCQ was omitted in those patients on Triple therapy (MTX+HCQ+SAAZ) (20). American College of Rheumatology recommends usage of DMARD monotherapy for naive RA patients with low to moderate disease activity ${ }^{12} .27$ cases were advised MTX monotherapy.

None of the patients who were already on DMARDs for RA were primarily diagnosed at our institute. Since they had no access to their treating physician or Rheumatologist, treatment was suggested through Teleconsultation. Although treatment of RA in these patients continued on the lines of recommended guidelines, counseling them to discontinue HCQ was difficult. This was also done after due consultation from the physician at our institute. However, the patients were also advised to contact their treating physician as and when the lockdown restrictions are lifted off.

RSI - Majority of the patients in our study presented with RSI of different types. Repetitive strain injury sometimes called as repetitive stress injury is usually the caused by overuse or repeated movements of a particular joint resulting in localized inflammation and a feeling of throbbing pain, weakness with 
inability to move the limb. Most common RSI was lateral epicondylitis of elbow (tennis elbow) followed by plantar fasciitis, strain of neck muscles, frozen shoulder, Achilles tendonitis, carpal tunnel syndrome, bursitis and trigger finger.

Diagnosis of RSI in these cases was based on detailed history to look for risk factors and video call based clinical examination eg. Pointing out the exact point of pain and Performing Wringing test for Lateral epicondylitis of elbow, pointing out the point of pain in plantar fasciitis and aggravation of pain on walking the initial few steps, tingling in the hand aggravated at night and numbness in median nerve distribution demonstrated by a hand diagram in Carpal tunnel syndrome, demonstration of triggering of finger.

Standard treatment of RSI was advised to all patients. This included hot/cold compresses, avoiding the strenuous activity, posture modification, splints/braces, strengthening exercises and anti-inflammatory drugs. The pictures of various splints/braces and Video recordings on exercises and posture adjustments were shared with the patients.

Four cases of plantar fasciitis who complained of chronic pain and no relief on conservative treatment were given appointments to visit hospital for local steroid injections.

Trauma - New guidelines have been established to manage acute trauma and Orthopaedics during this pandemic ${ }^{13,14}$. Orthopaedic trauma management generally requires a good infrastructure and manpower for efficient diagnosis and treatment (conservative or operative). Major limitations during Covid-19 lockdown were clinical examination and radiological investigations to arrive at a diagnosis of a fresh injury. Out of 93 trauma patients, 50 were either called to our hospital or visit nearby hospital for immediate care. POP slab/cast application was done in 16 patients who visited our hospital (Chart 1). Although Tele-consultation could not prevent the trauma affected patients from visiting the hospital, it did aid in the provisional diagnosis of the patients. After the initial visit, patients were asked to followup in Tele-consultation thereby establishing the Virtual Fracture Clinic ${ }^{15,16}$. The main functions of this clinic were to monitor the danger signs of POP cast/slab like loosening, breakage, signs of compartment syndrome, movements of the distal part, provide various treatment options and also to advice on the medications and revisits. Those requiring surgery were referred to centers where emergency operative procedures were being performed. No complications of fracture were encountered in any of the patient during the follow-up consultations.

Degenerative/Mechanical - Osteoarthritis knee and mechanical back pain are frequently diagnosed cases in Orthopaedic OPD. Out of 143 Cases 77 had OA knee and 66 cases had low back pain. Those with OA knee were advised anti-inflammatory drugs, life-style modifications and exercises through video calls. Those cases with severe OA knee requiring replacement; the surgery was postponed due to Covid19. Those with low back pain were evaluated with a thorough history to differentiate mechanical back strain from disc pathology. Few patients uploaded their MRI films and reports in the app which was 
accessible to the doctors. Those requiring neurological examination were called to hospital for consultation and accordingly treatment was advised. Postural modifications, anti-inflammatory drugs, hot compresses and avoiding strenuous activity was all that was required in majority of cases.

There were no cases of orthopaedic infection or tumor. The management would be equally challenging had there been any.

Initial total registrations for e-consultation were high. There was a gradual reduction in the number of strict lockdown red zones allowing the patients to access health care facility in nearby available dispensary and clinics. Hence e-consultation was less sought after and the daily registrations gradually decreased with time.

The major limitation of e-consultation during the lockdown period was investigating the patient by blood tests and scans ${ }^{17}$. Though the provision was made by AlIMS Mangalagiri to perform doorstep sample collections and deliver reports of blood investigations to nearby patients, performing scans were still limited. The poor quality of video and network related issue during consultation was another limitation which made difficult to arrive at a diagnosis and advise treatment. This limitation was overcome to certain extent by asking the patients to click photographs of affected body part especially in cases of trauma to look for wound and send it through WhatsApp for visualization. However, arriving at a diagnosis by a thorough history despite limited examination is the highlight of Tele-consultation, thereby signifying the importance of constructing a clinical history ${ }^{18}$.

\section{Conclusion}

Tele-consultation in Orthopaedics is a challenging entity in developing countries. Based on our experience, majority of Orthopaedic disorders were addressed through video consultation. Though Teleconsultation has limitations, it is nonetheless a convenient mean of delivering health care services to the public at large. Currently, Orthopaedic treatment through Video calls is not popular. With developing technology, this modality may very well be accepted. We strongly recommend the use of e-consultation services by the patients in the current scenario of Covid-19 scare, thus helping the nation fight the dreadful infection by preventing its spread. We at are working to improvise the Tele-Consultation services, resolving the drawbacks and limitations with innovative ideas so as to benefit a large population. Further studies are required to establish and make optimum use of Tele-consultation in the field of Orthopaedics.

\section{Declarations}

\section{Ethics:}

Institutional ethical clearance has been taken for the study

\section{References}


1. Ateriya N, Saraf A, Meshram VP, Setia P. Telemedicine and virtual consultation: The Indian perspective. Natl Med J India 2018;31:215-8

2. Ministry of health \& family welfare Telemedicine Practice Guidelines. Retrieved from www.mohfw.gov.in/pdf/Telemedicine.pdf

3. Mabiyan R, Covid-19 lockdown 0: Telemedicine in India to see continued growth. The Economics Times $16^{\text {th }}$ April,2020. www.health.economictimes.indiatimes.com/news/health-it/covid-19lockdown-2-0-Telemedicine-in-india-to-see-continued-growth/75172147 accessed 20 July 2020.

4. Chellaiyan, Vinoth $\mathrm{G}$ et al. "Telemedicine in India: Where do we stand?." Journal of family medicine and primary care vol. 8,6 (2019): 1872-1876

5. Van Galen LS, Car Telephone consultations. BMJ 2018;360:k1047.

6. Greenhalgh Trisha, Wherton Joe, Shaw Sara, Morrison Clare. Video consultations for covid-19 BMJ 2020; 368

7. "Tele-consultation." McGraw-Hill Concise Dictionary of Modern 2002. The McGraw-Hill Companies, Inc. 20 May. 2020 https://medicaldictionary.thefreedictionary.com/Tele-consultation

8. World Health Organization Strengthening the Health Systems Response to COVID-19. Retrieved from http://www.euro.who.int/data/assets/pdf_file/0007/436354/strengthening-health-systemsresponse-COVID-19-technical-guidance-1.pdf?ua=1

9. Dr GSCNV Prasad, Dr Gowtham ram, Dr Rama Mohan Desu, Dr Sreeram Sathish, Dr Arunkanth, Mrs Rajeswari, Shop Window of the Hospital: Service Delivery Perception by the Patients - An Experience at a Teaching INTERNATIONAL JOURNAL OF SCIENTIFIC RESEARCH : Volume-6 | Issue-12 |December-2017

10. Neha Sinha, Max Cornell, Benjamin Wheatley, Nicole Munley, Mark Seeley. Looking Through a Different Lens: Patient Satisfaction With Telemedicine in Delivering Pediatric Fracture Care. J Am Acad Orthop Surg Glob Res Rev. 2019 Sep; 3(9)

11. Taral Nagda. 2020 May 12. Clubfoot manipulation by parents. YouTube IN. https://www.youtube.com/watch?v=vgp3ld9rX9k

12. American College of Rheumatology. Rheumatoid Arthritis Guidelines; Available at https://www.rheumatology.org/Practice-Quality/Clinical-Support/Clinical-Practice Guidelines/R heumatoid-Arthritis

13. British Orthopaedic Association. Speciality guidelines, 2020. Available: https://www.boa.ac.uk/uploads/assets/ee39d8a8-9457-4533-9774e973c835246d/COVID-19BOASTs-Combined-v1FINAL.pdf [Accessed 12 Aug 2020].

14. NHS England and NHS Improvement Coronavirus. Specialty guides-Orthopaedic trauma, 2020. Available: https://www.england.nhs.uk/coronavirus/wp- content/uploads/sites/52/2020/03/C0070specialty-guide-major-trauma-clinical-guide-14 April- 2020.pdf

15. Logishetty K, Subramanyam Adopting and sustaining a Virtual Fracture Clinic model in the District Hospital setting - a quality improvement approach. BMJ Qual Improv Rep 2017;6.u220211.w7861. 
16. lyengar $\mathrm{K}$, Vaish $\mathrm{A}$, Toh $\mathrm{E}$, et al COVID-19 and remote consulting strategies in managing trauma and orthopaedics. Postgraduate Medical Journal 2020;96:438-439.

17. Greenhalgh T, Vijayaraghavan S, Wherton J, et al. Virtual online consultations: advantages and limitations (vocal) study. BMJ Open 2016;6

18. Deldar K, Bahaadinbeigy K, Tara SM. Teleconsultation and Clinical Decision Making: a Systematic Review. Acta Inform Med. 2016;24(4):286-292. doi:10.5455/aim.2016.24.286-292

\section{Tables And Chart}

Please see the Supplementary Files for Chart 1

Table 1: Demographic profile and patient characteristics

\begin{tabular}{|llll|}
\hline Parameter & Total Number & Fresh registrations & Follow-up registrations \\
\hline Total registrations included in study & $468(\mathrm{~N})$ & $375(\mathrm{n} 1)$ & $93(\mathrm{n} 2)$ \\
\hline Gender & & & \\
\hline Male & $205(43.8 \%)$ & $176(85.85 \%)$ & $39(14.15 \%)$ \\
\hline Female & $263(56.2 \%)$ & $209(79.46 \%)$ & $54(20.54 \%)$ \\
\hline Age Distribution & & & \\
\hline $0-12$ years & $21(4.48 \%)$ & 12 & 16 \\
\hline $13-30$ years & $121(25.8 \%)$ & 105 & 15 \\
\hline $31-50$ years & $157(33.54 \%)$ & 142 & 53 \\
\hline 51 and above years & $169(36.11 \%)$ & 116 & \\
\hline
\end{tabular}

Table 2: Diagnosis 


\section{Category}

Diagnosis

Number of

cases

Congenital $(0.26 \%)$

CTEV

$1 \quad 0.27 \%$

Developmental and Metabolic $(1.06 \%)$

Rickets

$4 \quad 1.07 \%$

Inflammatory (14.4\%)

Rheumatoid arthritis

$53 \quad 14.13 \%$

Ankylosing spondylitis

$1 \quad 0.26 \%$

Traumatic (24.8\%)

Soft tissue closed injury

$58 \quad 15.47 \%$

Soft tissue open injury

$3 \quad 0.80 \%$

Fracture/dislocation upper limb and

$25 \quad 6.67 \%$

chest

Fracture/dislocation lower limb and

Pelvis

Fracture spine

1

$0.27 \%$

Degenerative/Mechanical (38.13\%)

Knee OA

$77 \quad 20.53 \%$

Low back pain

$54 \quad 14.40 \%$

PIVD

$10 \quad 2.67 \%$

Spondylolisthesis

$2 \quad 0.53 \%$

RSI (20.53\%)

Lateral epicondylitis

Frozen shoulder

Dequervains tenosynovitis

Plantar fasciitis

Strain of neck muscles

Carpal tunnel syndrome

Achilles tendonitis
$16 \quad 4.26 \%$

$14 \quad 3.73 \%$

$5 \quad 1.33 \%$

$19 \quad 5.06 \%$

$10 \quad 2.67 \%$

$4 \quad 1.07 \%$

$5 \quad 1.33 \%$




\begin{tabular}{|llcc|} 
& Bursitis & 2 & $0.53 \%$ \\
\hline & Trigger finger & 2 & $0.53 \%$ \\
\hline Secondary arthritis $(0.87 \%)$ & Keinbocks disease & & \\
& AVN hip & 1 & $0.27 \%$ \\
\hline Infective & & 2 & $0.53 \%$ \\
\hline Tumor & & & \\
\hline
\end{tabular}

Figures 


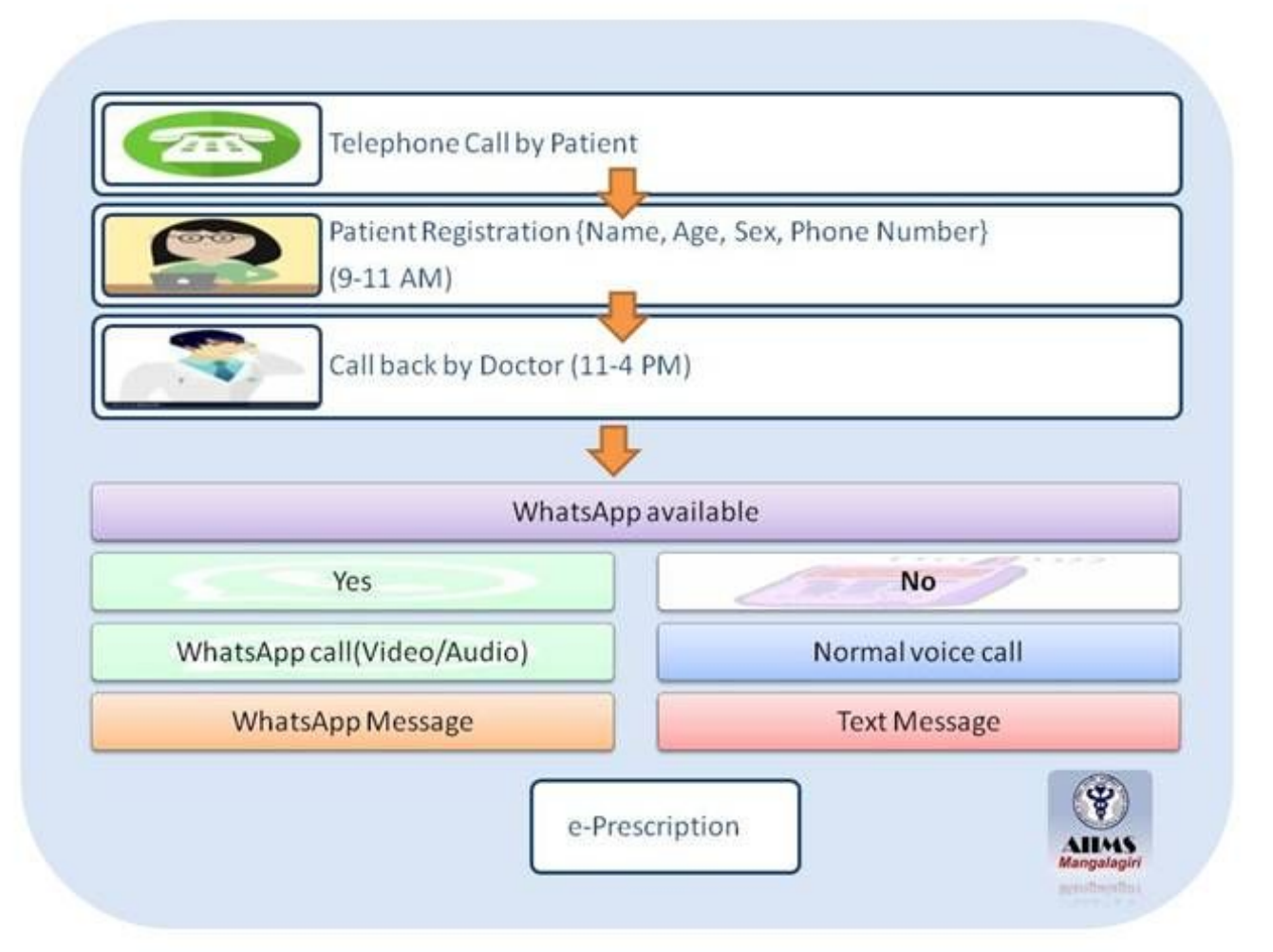

Figure 1

Call Based registration process 


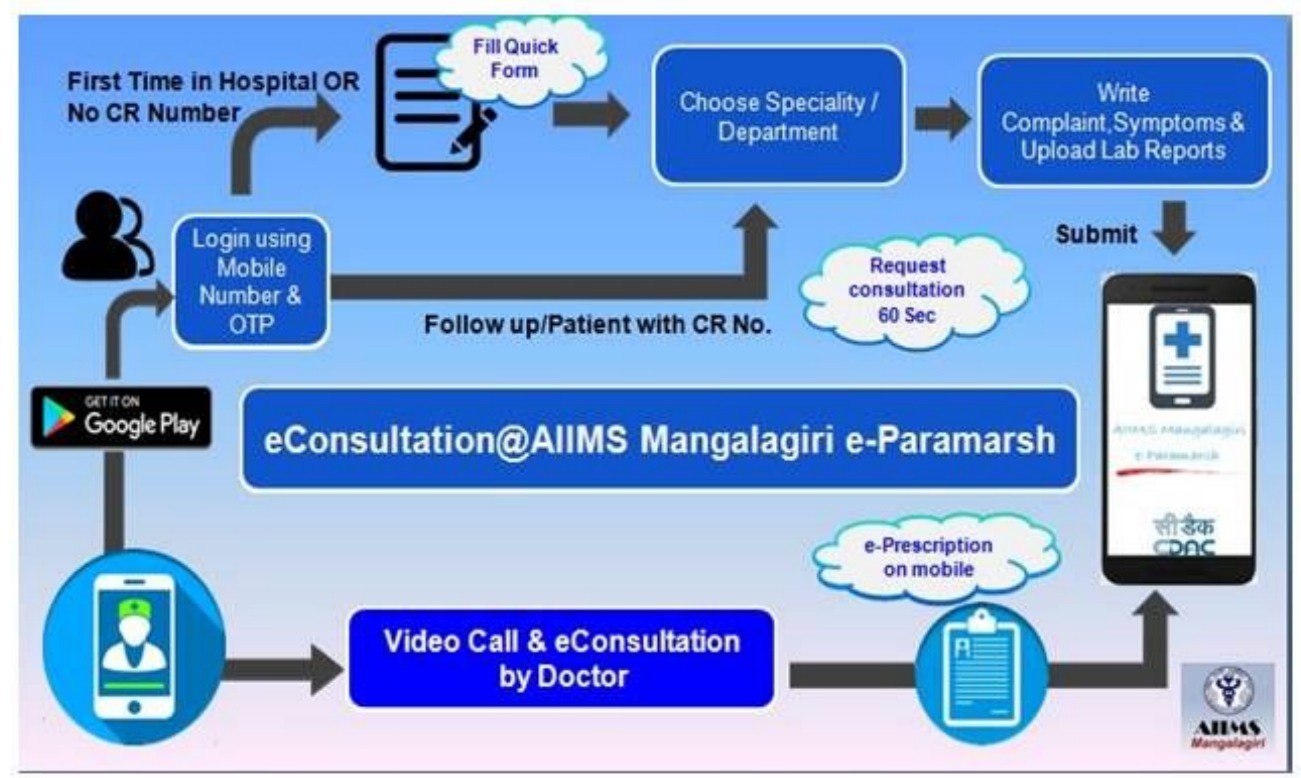

Figure 2

App Based registration process 


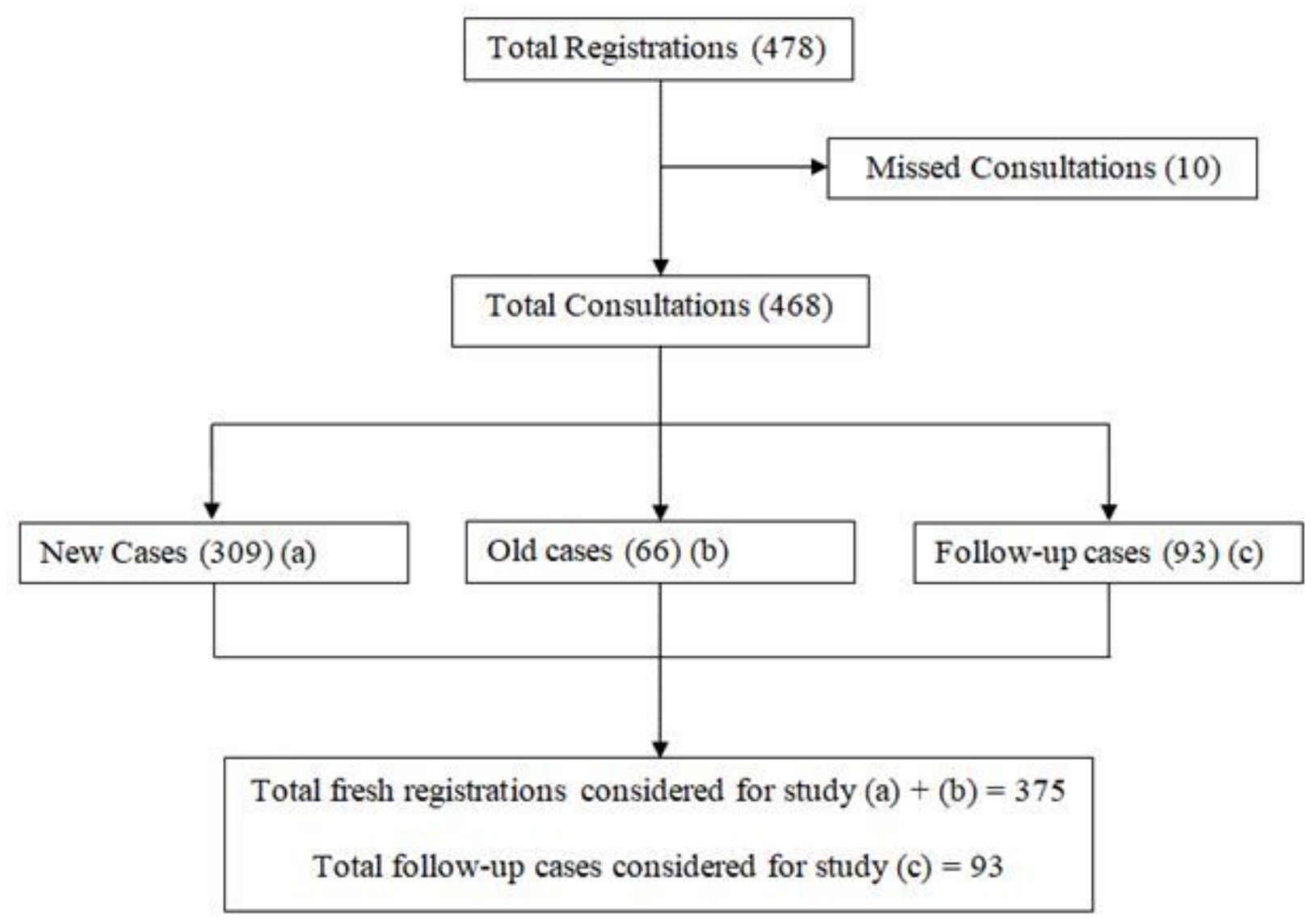

(a) Patients who consulted the doctor through Tele-consultation for the first time

(b) Patients who had consulted face-to-face prior to Covid-19 outbreak and the follow-up consultation was done via Tele-consultation

(c) Both new and old cases registered for their subsequent consultations via Teleconsultation

\section{Figure 3}

Flow chart for study sample selection

\section{Supplementary Files}

This is a list of supplementary files associated with this preprint. Click to download.

- Chart1.docx 